\title{
The Effect of Adaptation and Expectation on Initial Stances Prior to Automatic Postural Responses
}

\author{
Hiroshi Maejima ${ }^{1)}$, Chie Kamoda ${ }^{1)}$, TAtehito UedA ${ }^{1)}$, Hisato Sasaki ${ }^{1)}$, Sachiko Tanaka ${ }^{1)}$, \\ NAOHIKO KANEMURA ${ }^{1)}$, AKIRA MINEMATU ${ }^{1)}$, OSAMU YOSHIMURA ${ }^{1)}$ \\ 1) Institute of Health Sciences, Hiroshima University School of Medicine: \\ Kasumi 1-2-3, Minami-ku, Hiroshima 734-8551, Japan. \\ TEL +8182-257-5458,E-mail:maeji@hiroshima-u.ac.jp
}

J. Phys. Ther. Sci.

13: 93-98, 2001

\begin{abstract}
The purpose of this study was to clarify the effect of adaptation to and expectation of postural sway caused by perturbation of a platform on initial stances prior to postural response. Twenty healthy university students participated in this study. The joint angles of the ankle, knee, hip, and the neck in initial stance were measured. Adaptation to backward postural sway through repeated perturbations caused the body to tilt backward. However, expectation of backward body sway caused the body to tilt forward, compared with the initial stance when forward body sway was expected. It is suggested that expectation induces the initial stance to stabilize against postural sway mechanically prior to postural response. However, adaptation affects the efficiency of the postural response itself.

Key words: Postural sway, Three dimensional joint angle.
\end{abstract}

(This article was submitted Oct. 21, 2000, and was accepted Jan. 20, 2001)

\section{INTRODUCTION}

Falling due to unstable standing balance is considered to be one factor resulting in hip bone fractures, especially in elderly people, so the dynamic standing balance of humans is being given increasingly greater attention ${ }^{1-3)}$. The study of dynamic standing balance originated in Nashner's scheme in which postural response was examined against postural sway induced by a movable platform ${ }^{4,5)}$. Nashner's group found that two comprehensive muscle activation patterns are characteristic of this scheme. When a subject stands on a normal platform and postural disturbance is elicited by horizontal perturbation of the platform, muscle activity begins in the ankle joint muscles and radiates in a distal-to-proximal sequence on the same dorsal or ventral aspect. This pattern is termed ankle strategy. On the other hand, when a subject stands on a platform which is short in relation to foot length, the trunk and thigh muscles that are antagonistic to those used in ankle strategy are activated in the opposite proximal-to-distal sequence. This pattern is termed hip strategy. These responses are termed automatic postural response ${ }^{6,7)}$.

This scheme has been used extensively in the study of dynamic standing balance. In this experimental scheme, it is necessary to make the initial stances prior to postural sway consistent throughout the trials of one subject in order to quantify and qualify the postural response itself. Thus it is necessary to confirm that the Center of Pressure (COP) from the force plate and the postural alignment from the three dimensional analysis system is consistent throughout the trials.

It is supposed that the initial stance is influenced by adaptation to postural sway caused by repeated 


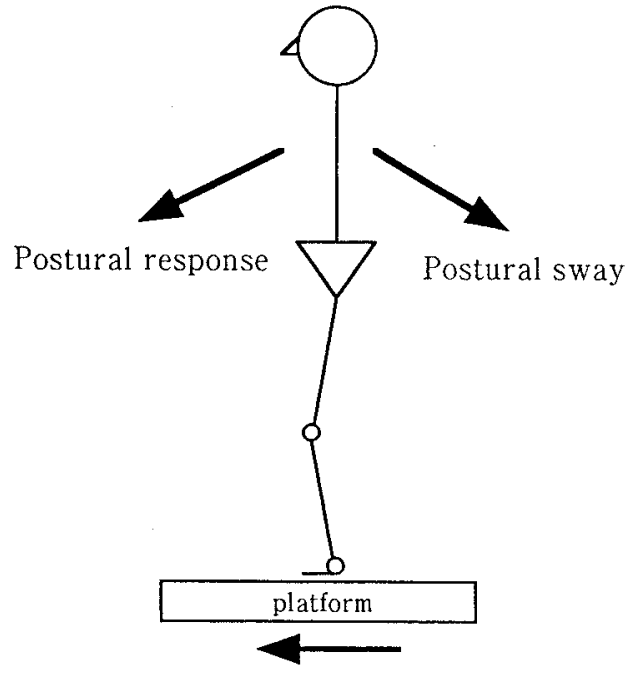

Perturbation of the platform

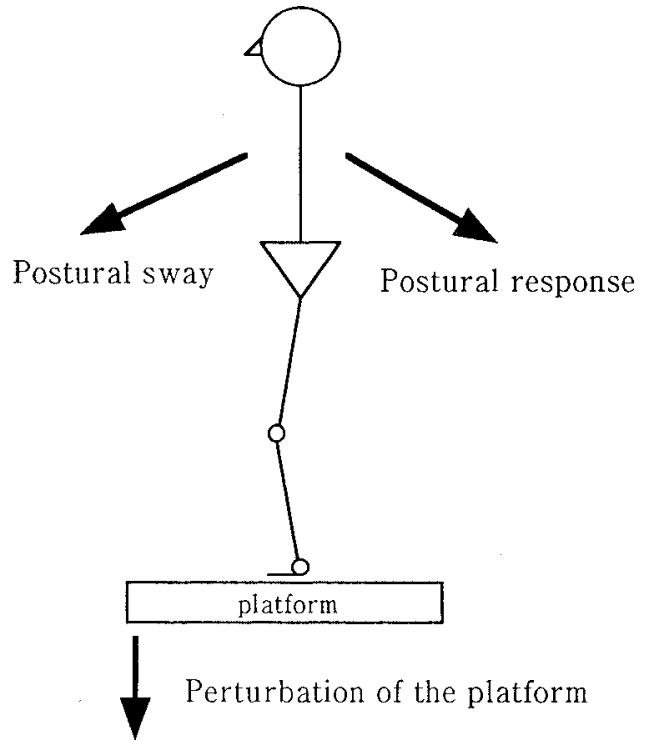

Fig. 1. Automatic postural response caused by two kinds of stimulous of the platform.

trials and expectation of a perturbation form which is characteristic in direction, amplitude and velocity of platform perturbation. On the other hand, from the point of view of improving standing balance and preventing falling, it is supposed that dynamic standing balance is concerned not only with the postural response itself but also with the process from the selection of an initial stance to the appearance of a postural response. It is supposed that the initial stance is a preparatory posture against postural sway which is selected through advanced inner factors like adaptation and expectation. The selection of an initial stance is therefore thought to be a part of the strategy for controlling dynamic standing balance.

The purpose of this study was to clarify the effect of the two inner factors of adaptation and expectation on initial stances prior to postural responses and to ascertain the significance of both factors in controlling postural balance.

\section{MATERIALS AND METHODS}

\section{Subjects}

Twenty healthy university students (age: $19.9 \pm$ 1.2) participated in this study.

\section{Apparatus}

The subjects stood with their eyes closed and their arms folded on a movable platform (our original portable perturbater; SAKAI Medical Co., Ltd.). The platform perturbations consisted of sudden forward perturbation which caused backward body sway and rotation which caused plantar flexion of the ankle and forward body sway. Seven emitting diodes were placed on the subject's body at the head of the lateral fifth metatarsal, lateral malleolous, lateral condyle (knee), lateral trochanter and chin, and the three-dimensional angles of the ankle, knee, hip and neck were measured with a three-dimensional movement analysis system (NORUMU KOUGAKU Co., Ltd). The emission of each diode was sampled at $83 \mathrm{~Hz}$ for $3000 \mathrm{~ms}$ (from $500 \mathrm{~ms}$ before the start of platform perturbation to $2500 \mathrm{~ms}$ after the start of platform perturbation, Fig. 1).

\section{Protocol}

Protocol 1 (effect of adaptation)

For the forward perturbation of the platform, three trials (the first session) were conducted following one practice trial. Three continuous trials (the middle session) were conducted following 12 practice trials, and lastly three trials (the last session) were conducted following 20 practice trials.

For the rotation of the platform, the same procedure was executed.

Protocol 2 (effect of expectation)

Two groups of trials were put in practice in turn. 
One group consisted of 3-5 trials with the same forward perturbation of the platform (forward group), and the other consisted of 3-5 trials with rotation of the platform (rotation group). Each group of trials was put into practice as follows: Forward group $\rightarrow$ Rotation group $\rightarrow$ Forward group $\rightarrow$ Rotation group $\rightarrow$ Forward group $\rightarrow$ Rotation group $\rightarrow$ one forward trial. Subjects attended each trial with the expectation that the stimuli of the next perturbation would be the same as that of the prior trial. The number of trials of each group was determined randomly, so subjects could not anticipate when the the stimuli of perturbation would change. The first trial of each group consisted of one in which subjects were unable to anticipate the stimuli of perturbation for the group, and the last trial of each group consisted of one in which subjects could anticipate the stimuli of perturbation. Three trials for each situation (the first forward perturbation, the last forward perturbation, the first rotation and the last rotation) were conducted.

The position of the feet of the standing subject was marked on the platform, and it was checked prior to the perturbation whether the position of the feet was consistent with that of previous trials. Subjects looked at a mark in front of them to adjust their neck angle to that of previous trials and closed their eyes. Subjects were required to maintain the same initial stance as in the previous trial. Each trial was preceded by a $30 \mathrm{sec}$. intermission in both protocols.

\section{Data analysis}

The joint angles of the foot, knee, hip and the neck were measured for $300 \mathrm{msec}$. from $400 \mathrm{msec}$. to $100 \mathrm{msec}$. prior to the onset of perturbation of the platform. The average values of three trials in the same situation were measured for each protocol.

The data were not normally distributed, so the Friedman test was used to clarify the effect of adaptation among the three groups, and Wilcoxon's rank sum test was used between the two groups (alpha $=0.05$ ). Wilcoxon's rank sum test was also used to clarify the effect of expectation between the two groups (alpha $=0.05$ ).

\section{RESULTS}

\section{The effect of adaptation to perturbation on initial stance}

Adaptation to forward perturbation of the

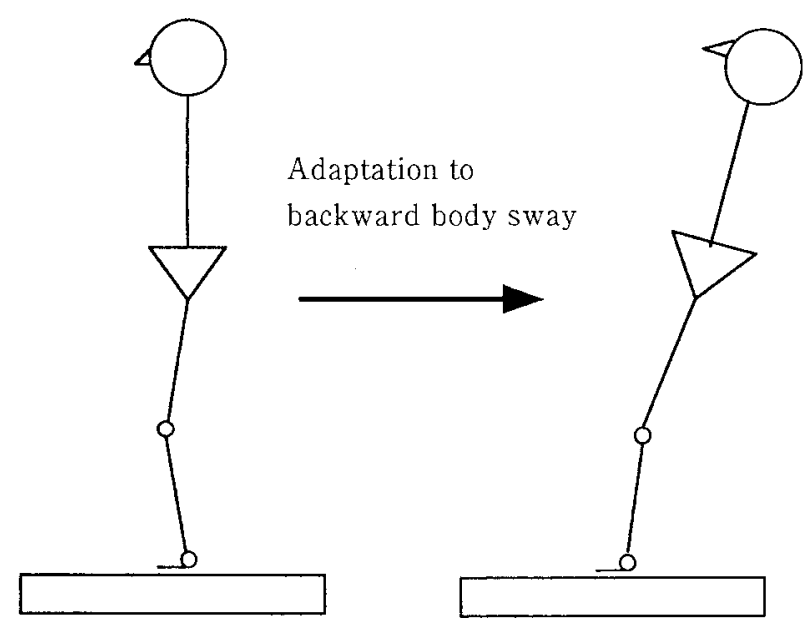

Fig. 2. Effect of adaptation to backward body sway caused by forward perturbation of the platform on initial stance.

\section{platform}

As an adaptation to forward perturbation of the platform, the joint angle of the ankle in the initial stance significantly changed toward dorsi-flexion $(p<0.01)$. There was no significant change in the joint angle of the knee, the hip and the neck (Table 1A). As a result of adaptation, the body tilted backward around the ankle, which caused instability against backward sway of the body caused by forward perturbation of the platform (Fig. 2).

Adaptation to rotation of the platform

There was no significant change in any joint angle in the initial stance through repeated trials of rotation of the platform. However, the joint angle of the hip tended to increase toward extension $(\mathrm{p}<0.06$, Table 1B).

\section{The effect of expectation of perturbation on ini- tial stance}

When subjects expected forward perturbation of the platform which causes backward sway of the body, the angle of the ankle increased significantly toward plantar-flexion, the angle of the knee joint increased significantly toward extension and the angle of the hip joint decreased toward flexion, compared with the initial stance when subjects expected rotation of the platform which causes forward sway of the body $(\mathrm{p}<0.001, \mathrm{p}<0.001$ and $\mathrm{p}<0.01$, Table 2).

Accordingly, the initial stance tilted forward 
Table 1A. Change of joint angles in initial stance caused by adaptation to forward perturbation of the platform

\begin{tabular}{lrrc}
\hline & \multicolumn{1}{c}{ First } & \multicolumn{1}{c}{ Middle } & \multicolumn{1}{c}{ Last } \\
\hline Ankle (deg.) & $85.7 \pm 5.1$ & $86.2 \pm 4.6$ & $86.6 \pm 4.9 *$ \\
Knee (deg.) & $172.9 \pm 4.9$ & $172.9 \pm 4.9$ & $172.4 \pm 4.9$ \\
Hip (deg.) & $158.7 \pm 3.1$ & $158.1 \pm 3.8$ & $158.5 \pm 3.9$ \\
Neck (deg.) & $53.3 \pm 4.2$ & $54.1 \pm 3.2$ & $53.8 \pm 3.8$ \\
\hline
\end{tabular}

$* \mathrm{p}<0.05$.

Table 1B. Change of joint angles in initial stance caused by adaptation to plantar rotation of the platform

\begin{tabular}{lrrr}
\hline & \multicolumn{1}{c}{ First } & \multicolumn{1}{c}{ Middle } & \multicolumn{1}{c}{ Last } \\
\hline Ankle (deg.) & $88.0 \pm 5.3$ & $88.0 \pm 5.4$ & $87.7 \pm 5.6$ \\
Knee (deg.) & $171.2 \pm 5.1$ & $171.9 \pm 5.2$ & $171.6 \pm 5.2$ \\
Hip (deg.) & $159.1 \pm 3.8$ & $159.5 \pm 3.7$ & $159.5 \pm 3.7$ \\
Neck (deg.) & $53.7 \pm 3.9$ & $53.9 \pm 3.8$ & $54.4 \pm 4.1$ \\
\hline
\end{tabular}

when backward postural disturbance was expected, compared with the initial stance when forward postural disturbance was expected. Conversely, the initial stance tilted backward when forward postural disturbance was expected, compared with the initial stance when backward postural disturbance was expected (Fig. 3).

\section{DISCUSSION}

The latency in the electromyogram (EMG) of agonists in the lower legs of the postural response induced by perturbation of platform was shorter than $100 \mathrm{~ms}$. Horak et al. calls this response an automatic postural response. Forward perturbation of the platform causes backward postural sway. Against this postural sway, induced activation of agonists like tibialis anterior (TA) and quadriceps femoris (QUAD) return the center of gravity (COG) of the body forward. On the other hand, plantar rotation of the platform causes forward postural sway. Against this postural sway, induced activation of agonists like triceps surae (TS) and hamstrings (HAM) return COG of the body backward $^{8)}$ (Fig. 1).

\section{The effect of adaptation to perturbation on initial stance}

In adaptation to forward perturbation of the platform, the initial stance tilted backward unexpectedly, which is a more unstable posture against postural sway. This means adaptation to
Table 2. Change of joint angles in initial stance caused by expectation

\begin{tabular}{lcc}
\hline & $\begin{array}{c}\text { Expect forward } \\
\text { perturbation of } \\
\text { the platform }\end{array}$ & $\begin{array}{c}\text { Expect plantar } \\
\text { rotation of } \\
\text { the platform }\end{array}$ \\
\hline Ankle (deg.) & $86.4 \pm 5.4$ & $87.2 \pm 5.7^{* *}$ \\
Knee (deg.) & $172.6 \pm 4.7$ & $171.1 \pm 5.2^{* *}$ \\
Hip (deg.) & $158.6 \pm 4.1$ & $159.7 \pm 4.3^{* *}$ \\
Neck (deg.) & $54.6 \pm 4.2$ & $54.6 \pm 4.3$ \\
\hline
\end{tabular}

$* * \mathrm{p}<0.001$.

postural perturbation does not affect postural strategy the goal of which is to decrease postural perturbation mechanically. On the other hand, repeated trials of rotation of the platform did not affect the initial stance. This suggests that the length of the foot as a moment arm supports the lower legs and helps reduce activity of the agonists inducing a larger moment around the ankle. Accordingly there is no need for a significant change in the initial stance for this perturbation.

So far it has been reported that repeated experience of postural sway induced by platform perturbation reduces postural sway and muscle activities in postural response under a consistent initial stance. Horak et al. reported that, according to adaptation, the activity of the antagonist which interferes with torque around the ankle was inhibited and that the activity of the agonist also decreased ${ }^{8,9)}$. Hansen et al. also reports that there are two components that contribute to the response reduction. One component is an initial reduction between the first trial and subsequent trials, which is due to habituation of a startle-like response. The other is a second reduction, which is a more gradual adaptation over more than ten trials ${ }^{10)}$.

In previous studies, though the initial stances were corrected to be consistent, it is supposed that the static muscle tone of the agonists and antagonists changes in the consistent initial stance, which may have an effect on postural response. In our previous study in which initial stances were not corrected as in this study, not only the activity of the agonists decreased but also the latency of the agonists became longer in the electromyogram ${ }^{11}$ ) (EMG). This result in latency was not consistent with Horak's report in which the latency of the agonists did not change in corrected initial stances ${ }^{10)}$. The difference in the initial stance as a 


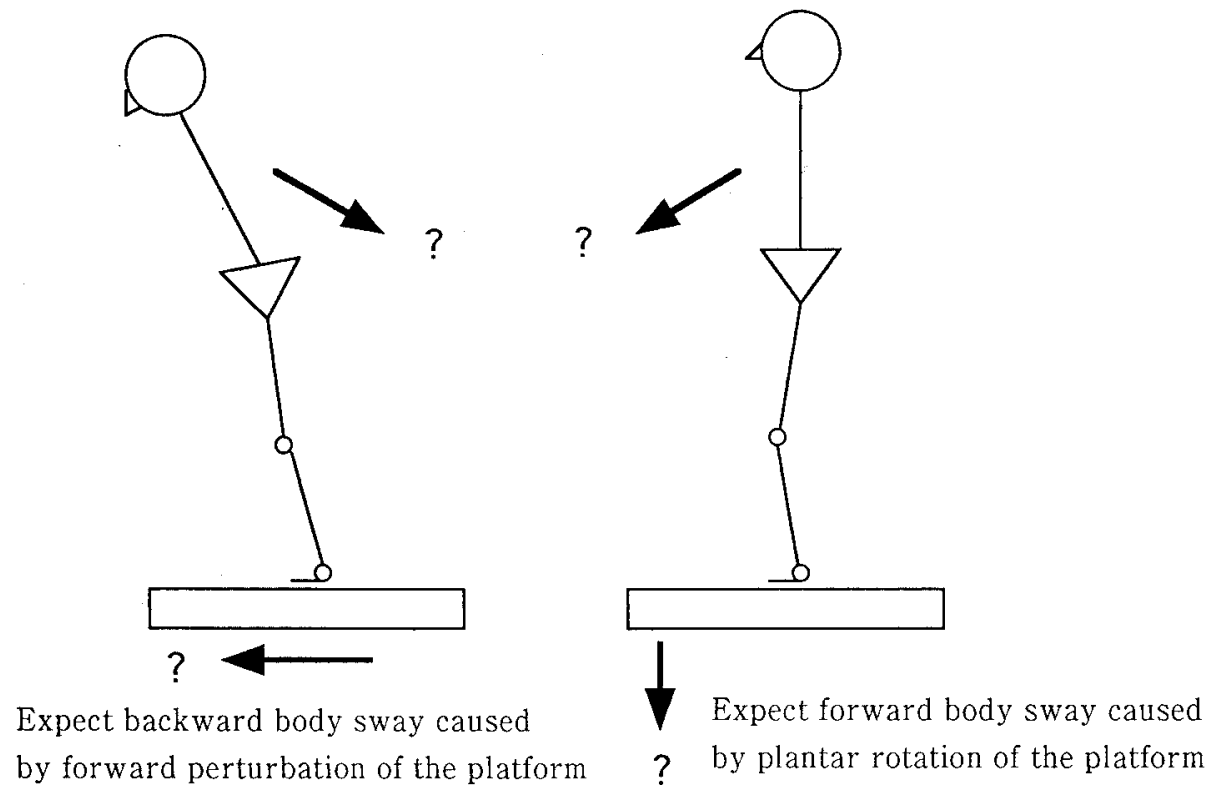

Fig. 3. Effect of expectation of body sway on initial stance.

preparatory posture and the distribution of muscle tone as a correction of initial stance were suggested as factors influencing this different result. In these previous studies, it was thought that adaptation was more closely related to the efficiency of postural response itself than the reduction of postural perturbation due to mechanical support.

\section{The effect of expectation of perturbation on ini- tial stance}

When subjects expected backward postural sway caused by forward perturbation of the platform, the initial stance tilted forward, and when subjects expected forward postural sway caused by rotation of the platform, the initial stance tilted backward. Forward movement of the COG in the initial stance inhibits backward disturbance of $\mathrm{COG}$ mechanically, and backward movement of COG in the initial stance also inhibits forward disturbance of COG mechanically. This result indicates that the expectation of stimuli of perturbation has an effect on the strategy, the goal of which is to reduce postural perturbation mechanically.

On the effect of expectation of the stimuli of perturbation, Diener et al. reports that cognition of the stimuli of perturbation does not affect postural response itself ${ }^{12}$. Macpherson et al. also reported that expectation did not affect postural response in an experiment using cats ${ }^{13)}$. Hansen et al. did not find that the stimuli of a previous perturbation had an effect on postural response in the next trial ${ }^{10)}$. These reports suggest that expectation of the form of perturbation has the effect of increasing mechanical stability in the initial stance before perturbation rather than in the postural response itself in the phase after perturbation.

\section{CONCLUSION}

Expectation of postural sway affects the mechanism in which postural perturbation is inhibited mechanically in the initial stance. It was conjectured that adaptation to postural sway affected the mechanism in which postural perturbation is inhibited through improvement of the efficiency of the postural response itself. The results also suggest that instability in the initial stance due to adaptation (for example, plantar flexion for backward postural sway) can stretch the agonists and give adequate muscle tone for efficient muscle contraction and flexibility for postural response.

\section{REFERENCES}

1) Maki BE et al.: A prospective study of posture balance and risk of falling inambulatory and independent elderly population. J Gerontol 1994; 49: M27-M84.

2) Hasiao ET, Robinovitch $\mathrm{SN}$ : Common protective 
movements govern unexpected falls from standingheight. J Biomechanics 1998, 31: 1-9.

3) Cumming Sr et al.: Fall frequency and characteristic and risk of hip fractures. American Geriatrics Society, 1994, 42: 774-778.

4) Nashner LM et al.: Fixed patterns of rapid postural responses among leg muscles during stance. Exp Brain Res, 1977, 30: 13-24.

5) Nashner LM et al.: Organization of rapid responses to postural and locomototor -like perturbation of standing man. Exp Brain Res, 1979, 36: 463-476.

6) Horak FB, Nashner LM: Two distinct strategies for stance posture control: adaptation to altered support surface configurations. Soc Neurosci Abstr, 1983, 10: 65 .

7) Horak FB et al.: Postural orientation and equilibrium. In: Smith JL, ed. Handbook of Physiology, Section12: Oxford Univ Press Inc., 1996, 255-292.

8) Horak FB et al.: Central programming of postural movements: adaptation to altered support-surface configurations. J Neurophysiol, 1986, 55-6: 13691381.

9) Horak FB et al.: Influence of central set on human postural responses. J Neurophysiol, 1989, 62-4: 841853.

10) Hansen $P D$ et al.: Postural responses to changing task conditions. Exp Brain Res, 1988, 73: 626-636.

11) Maejima $H$ : The effect of adaptation and footwear conditions on dynamic standing balance. PROCEEDINGS: 13th International Congress of the WCPT. 1999, 455.

12) Diener $\mathrm{HC}$ et al.: Direction and amplitude precuing has no effect on automatic postural responses. Exp Brain Res, 1991, 84: 219-223.

13) Mcpharson JM et al.: The force constraint strategy for stance is independent of prior experience. Exp Brain Res, 1994, 101: 397-405. 